\title{
Resultados relevantes del Congreso Europeo de Cardiología 2018 en Munich: hallazgos sorpresivos del estudio FUTURE
}

\author{
Highlights results from European Congress of Cardiology 2018: \\ the FUTURE trial results
}

Revista Argentina de Cardioangiología Intervencionista 2018;9(3):131-132. Doi: 10.30567/RACI/20183/0131-0132

En el congreso europeo de cardiología recientemente finalizado se presentaron los resultados del estudio randomizado (RCT) FUTURE (FUnctional Testing Underlaying REvascularization)1.

El estudio se diseñó como de superioridad para comparar en pacientes de múltiples vasos el tratamiento con angioplastia guiada, cirugía coronaria $(\mathrm{CABG})$ y/o tratamiento médico. La estrategia se seleccionaba por tests funcionales durante el cateterismo, específicamente FFR (fractional flow reserve) o guiadas por angiografía y test funcionales.

Este RCT involucró a 21 centros de Francia con experiencia en FFR, y el primer autor y presentador del estudio fue investigador de los estudios FAME y un pionero del método en Francia ${ }^{2,3}$.

La FFR está dentro de las guías de tratamiento en aquellos pacientes con enfermedad de múltiples vasos sometidos a PCI (IIa B) 4 .5.

En los RCT, menos del 50\% de los pacientes con enfermedad de multiples vasos candidatos a PCI tienen enfermedad funcionalmente severa de múltiples vasos, y algo similar está reportado en los registros ${ }^{2,3,6}$.

El estudio, que fue un non industry sponsor study, abarcó a 21 centros en Francia, y el punto final primario compuesto era muerte de toda causa, infarto, accidente cerebrovascular (ACV) y nuevas revascularizaciones.

La hipótesis era que la rama de FFR iba a reducir un $30 \%$ de eventos al año.

Todos los pacientes tenían enfermedad de múltiples vasos definida como lesión $>50 \%$ con compromiso de la arteria descendente anterior.

Los pacientes presentaban angina estable y/o estaban estabilizados al momento de la randomización y la muestra de la población era de alrededor de 864 pacientes en cada grupo.

Sin embargo, el estudio fue suspendido precozmente por el comité de seguridad cuando el reclutamiento alcanzó los 937 pacientes por un exceso de mortalidad en el grupo manejado por FFR ( $p=0,038$; IC95\%: 1,05-5,43).

$\mathrm{Al}$ momento de la suspensión del estudio, las características basales demográficas, clínicas y angiográficas fueron similares a excepción de la incidencia de ACV previo, que fue estadísticamente mayor en el grupo control. Los pacientes guiados por FFR tuvieron una significativa menor incidencia de angioplastia y mayor incidencia de tratamiento médico comparado con el grupo control $(\mathrm{p}=0,002)$. El número de $\mathrm{CABG}$ y $\mathrm{PCI}$ ad hoc fue similares en ambos grupos.

Como dijimos, hubo una significativa mayor incidencia de muertes en el grupo FFR, lo que llevó al comité de seguridad a suspender el reclutamiento y parar el estudio.

$\mathrm{Al}$ momento de la presentación, este trabajo generó grandes controversias, dado que la FFR está en las guías europeas y muy difundida como método de elección para individualizar lesiones de diferente riesgo, considerándose casi como el gold standard al momento de la angioplastia en enfermedad de múltiples vasos.

No voy a poner en consideración en este breve resumen si el comité de seguridad cometió un error al suspender prematuramente el estudio, aunque la mortalidad fue más del doble en el grupo FFR $(3,7 \% v s .1,5 \%)$, y solamente me referiré a los hallazgos realizados.

$\mathrm{Al}$ momento de interrumpir el reclutamiento de los pacientes, el estudio quedó con 937 enfermos, 467 y 460 en los grupos control y FFR, respectivamente.

Las características basales mostraron algunas diferencias, como mayor historia de ACV previo en el grupo control $(\mathrm{p}=0,03)$. De igual manera, y aunque la incidencia de diabéticos fue similar $(\mathrm{p}=0,9)$ al alta hospitalaria, en el grupo FFR un significativo mayor número de pacientes fue tratado con insulina ( $\mathrm{p}=0,03)$, aunque el número de pacientes con requerimiento de insulina al alta fue muy pequeño $(2,2 \%)$.

Los resultados del punto final primario compuesto a uno y dos años fue similar entre ambos grupos, descartando la hipótesis inicial de la superioridad de la FFR para guiar revascularización en pacientes clínicamente estables y en presencia de múltiples vasos.

Como se dijo, hubo una mayor incidencia de muerte en el grupo FFR y una tendencia a mayor ACV ( $p=0,06)$ en el grupo control; se recuerda que este último grupo había tenido basalmente significativas diferencias en la historia de $\mathrm{ACV}$ previo.

De manera que podemos enumerar como limitaciones del estudio: primero, que basalmente hay algunas diferencias, balanceadas, entre ambos grupos; segundo, que el estudio no tendría potencia estadística para demostrar superioridad con el tamaño de la muestra; tercero, un exceso de PCI ad hoc; cuarto, que la mortalidad en el grupo FFR a 30 días fue largamente mayor que la observada en los estudios $\mathrm{FAME}^{3,6-7}$ y que no debería haber sido prematuramente detenido el ensayo etc., etc. 
Sin embargo, el hallazgo más importante no es la diferencia de mortalidad, ya que entraríamos en una discusión que escapa al propósito de este Editorial sobre la calidad técnica de los operadores de FFR, que, como dijimos antes, al menos la mayoría eran expertos en el procedimiento. A mi entender, el punto final primario, que es la suma de los eventos adversos combinados a 1 y 2 años (alrededor de 14 y $28 \%$ en ambos grupos, respectivamente) fue prácticamente idéntica y el estudio con su actual tamaño de la muestra tiene poder para demostrar la no inferioridad de una estrategia sobre la otra; esto, que parece sacado de contexto con las actuales guías ${ }^{4}$, no nos debería extrañar dado que es parte de la historia de PCI en enfermedad de múltiples vasos ${ }^{8-13}$.

El estudio incorporó lesiones intermedias en la estrategia de revascularización, por lo cual es de suponer que la rama guiada por angiografía o grupo control en su gran mayoría no incluyó estas lesiones en la estrategia de la PCI y ese conducta fue la que guió en el pasado todos los RCT de múltiples vasos entre PCI y CABG ${ }^{8-13}$.

Dichos estudios, como el que analizamos acá, involucraban a pacientes con angina crónica estable o estabilizados clínicamente donde la PCI fue guiada por estudios funcionales previos y donde se trataban solo lesiones angiográficamente severas definidas como $>70 \%$. En estos RCT la revascularización "completa anatómica" obtenida con CABG al año de seguimiento tuvo la misma incidencia de eventos que aquella revascularización "funcionalmente completa" obtenida con la $\mathrm{PCI}^{8-9}$.

Más recientemente, este pensamiento se aplicó para construir scores de riesgo basales y residuales post-PCI que también se asociaron con una baja incidencia de eventos adversos al follow up en pacientes similares a los presentados por el estudio FUTURE ${ }^{14-16}$.

Por otra parte, la valoración del grado de estenosis con FFR tiene bien descripta varias limitaciones, incluyendo una demostrada ausencia de beneficio en pacientes sometidos a $\mathrm{CABG}{ }^{17}$.

En resumen, y más allá de las limitaciones del estudio que motivó este Editorial ${ }^{1}$, el hallazgo de que en pacientes con múltiples vasos y con angina estable o estabilizados clínicamente los recursos terapéuticos, ya sean PCI, CABG o tratamiento médico, puedan ser utilizados correctamente mediante el análisis clínico y la valoración angiográfica de su anatomía no nos debería sorprender, ya que es un recurso que los cardiólogos y cardiólogos intervencionistas lo ponemos en práctica con éxito desde hace más de 25 años.

Alfredo E. Rodríguez Editor en Jefe de RACI

\section{BIBLIOGRAFÍA}

1. Rioufol G, Roubille F, Perret T, et al on behalf of the FUTURE trial investigators, France NCT01881555 FUnctional Testing Underlying Revascularization The FUTURE trial ESC Munich, Germany, August 2018.

2. Van Belle E, Rioufol G, Pouillot C, et al; Investigators of the Registre Français de la FFR-R3F. Outcome impact of coronary revascularization strategy reclassification with fractional flow reserve at time of diagnostic angiography: insights from a large French multicenter fractional flow reserve registry. Circulation. 2014 Jan 14;129(2):173-85.

3. Tonino PA, Fearon WF, De Bruyne B, et al. Angiographic versus functional severity of coronary artery stenoses in the FAME study fractional flow reserve versus angiography in multivessel evaluation. J Am Coll Cardiol 2010;55(25):2816-21.

4. Authors/Task Force members, Windecker S, Kolh P, Alfonso F et al. 2014 ESC/EACTS Guidelines on myocardial revascularization: The Task Force on Myocardial Revascularization of the European Society of Cardiology (ESC) and the European Association for Cardio-Thoracic Surgery (EACTS) Developed with the special contribution of the European Association of Percutaneous Cardiovascular Interventions (EAPCI). Eur Heart J. 2014 Oct 1;35(37):2541-619.

5. Lotfi A, Davies JE, Fearon WF, Grines CL, Kern MJ, Klein LW. Focused update of expert consensus statement: Use of invasive assessments of coronary physiology and structure: A position statement of the society of cardiac angiography and interventions. Catheter Cardiovasc Interv. $2018 \mathrm{Jul}$ 3. doi: $10.1002 / \mathrm{ccd} .27672$

6. Fearon WF, Nishi T, De Bruyne B, et al; FAME 2 Trial Investigators.Clinical Outcomes and Cost-Effectiveness of Fractional Flow Reserve-Guided Percutaneous Coronary Intervention in Patients With Stable Coronary Artery Disease: Three-Year Follow-Up of the FAME 2 Trial (Fractional Flow Reserve Versus Angiography for Multivessel Evaluation). Circulation. 2017 Nov.

7. Xaplanteris P, Fournier S, Pijls NHJ, et al; FAME 2 Investigators. Five-Year Outcomes with PCI Guided by Fractional Flow Reserve. N EnglJ Med. 2018 Jul 19;379(3):250-9.

8. Faxon D. Galilli K, Jacobs $A$, et al . The degree of revascularization and outcome after multivessel coronary angiography. Am Heart J 1992; 123 :854-9.

9. Rodriguez A, Boullon F, Perez Baliño N, et al. Argentine randomized trial of percutaneous transluminal coronary angioplasty versus coronary artery bypass surgery in multivessel disease (ERACI): in-hospital results and 1-year follow-up. J Am Coll Cardiol 1993; 33:1060-7.

10. King SB III, Lembo NJ, Weintraub WS, et al. A randomized trial comparing coronary angioplasty with coronary bypass surgery. N Engl J Med 1994; 331:1044-50.

11. CABRI Trial participants. Coronary Angioplasty vs. Bypass Revascularization Investigation (CABRI) results during the first year. Lancet 1995; 346:1179-83.

12. Hamm CW, Reimers J, Ischinger T, et al. A randomized study of coronary angioplasty compared with bypass surgery in patients with symptomatic multivessel coronary artery disease. N Engl J Med 1994;331:1037-43.

13. Pocock S, Henderson R, Rickards A, et al. Meta-analysis of randomized trials comparing coronary angioplasty with bypass surgery. Lancet 1995;346:1184-9.

14. Rodríguez AE, Fernández-Pereira C, Mieres J, Santaera O, Antoniucci D; ERACIIV investigators. Modifying angiographic syntax score according to PCl strategy: lessons learnt from ERACI IV Study. Cardiovasc Revasc Med. 2015 Oct-Nov; 16(7):418-20.

15. Haiek C, Fernández-Pereira C, Santaera O et al Second vs. First generation drug eluting stents in multiple vessel disease and left main stenosis: Twoyear follow-up of the observational, prospective, controlled, and multicenter ERACI IV registry. Catheter Cardiovasc Interv. 2017 Jan;89(1):37-46.

16. Rodriguez AE, Fernández-Pereira C, Mieres J, et al. Lowering risk score profile during $P C l$ in multiple vessel disease is associated with low adverse events: The ERACI risk score.Cardiovasc Revasc Med. 2018 Feb 13. pii: S1553-8389(18)30014-9. doi: 10.1016/j.

17. Toth G, De Bruyne B, Casselman F, et al. Fractional flow reserve-quided versus angiography guided coronary artery bypass graft surgery. Circulation 2013;128:1405-11. 